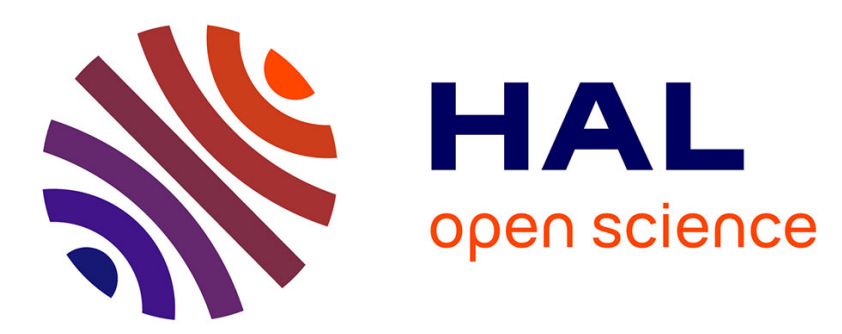

\title{
Epitaxie par jets moléculaires de In0,53Ga0,47As et de InP sur substrats de InP
}

\author{
M. Lambert, D. Huet
}

\section{To cite this version:}

M. Lambert, D. Huet. Epitaxie par jets moléculaires de In0,53Ga0,47As et de InP sur substrats de InP. Revue de Physique Appliquée, 1983, 18 (12), pp.757-761. 10.1051/rphysap:019830018012075700 . jpa-00245143

\section{HAL Id: jpa-00245143 https://hal.science/jpa-00245143}

Submitted on 1 Jan 1983

HAL is a multi-disciplinary open access archive for the deposit and dissemination of scientific research documents, whether they are published or not. The documents may come from teaching and research institutions in France or abroad, or from public or private research centers.
L'archive ouverte pluridisciplinaire HAL, est destinée au dépôt et à la diffusion de documents scientifiques de niveau recherche, publiés ou non, émanant des établissements d'enseignement et de recherche français ou étrangers, des laboratoires publics ou privés. 


\title{
Epitaxie par jets moléculaires de $\mathrm{In}_{0,53} \mathrm{Ga}_{0,47} \mathrm{As}$ et de InP sur substrats de InP
}

\author{
M. Lambert et D. Huet \\ Laboratoires de Marcoussis, Centre de Recherches de la C.G.E., route de Nozay, 91460 Marcoussis, France
}

(Reçu le 27 juillet 1983, accepté le 29 août 1983)

\begin{abstract}
Résumé. - Des couches de $\operatorname{In}_{0,53} \mathrm{Ga}_{0,47} \mathrm{As}$ de haute pureté, accordées avec le substrat de InP, ont été épitaxiées par la technique des jets moléculaires (E.J.M.). Les concentrations d'impuretés résiduelles sont de l'ordre de $10^{15} \mathrm{~cm}^{-3}$, avec des mobilités électroniques allant jusqu'à $47000 \mathrm{~cm}^{2} / \mathrm{V}$.s à $77 \mathrm{~K}$. La bonne qualité des couches est obtenue en contrôlant la préparation du substrat, la température du substrat, la pression d'arsenic et le recuit des produits sources. La caractérisation des couches est faite par diffraction de rayons X, effet Hall et photoluminescence à basse température. Les dopages de type nà l'étain et de type $\mathrm{p}$ au béryllium sont contrôlés de $10^{16} \mathrm{~cm}^{-3}$ à $10^{19} \mathrm{~cm}^{-3}$. Enfin, nos premiers résultats d'E.J.M. de InP sont présentés.
\end{abstract}

\begin{abstract}
High purity $\mathrm{In}_{0.53} \mathrm{Ga}_{0.47}$ As layers, lattice matched to InP, have been grown by molecular beam epitaxy (M.B.E.). Residual impurities levels are in the range of $10^{15} \mathrm{~cm}^{-3}$ with electron mobilities up to $47000 \mathrm{~cm}^{2} / \mathrm{V}$.s at $77 \mathrm{~K}$. The high quality of the epilayers is obtained by controlling the substrate preparation, the substrate temperature, the arsenic pressure and the baking of the source materials. The layers are characterized by X-ray diffraction, Hall effect and low temperature photoluminescence. Doping levels are controlled between $10^{16} \mathrm{~cm}^{-3}$ and $10^{19} \mathrm{~cm}^{-3}$, with thin for n-type and beryllium for p-type. Our first results on M.B.E. of InP are also reported.
\end{abstract}

\section{Introduction.}

Les alliages ternaires $\operatorname{In}_{1-x} \mathrm{Ga}_{x} \mathrm{As}$ offrent de grandes possibilités pour l'élaboration de dispositifs optoélectroniques [1] ou hyperfréquence [2].

La réalisation de tels dispositifs requiert avant toute chose la maîtrise de la croissance de matériaux de bonnes qualités cristalline et électronique, caractérisés en particulier par une faible densité de défauts ponctuels et étendus et un faible dopage résiduel typiquement inférieur ou égal à $10^{15} \mathrm{~cm}^{-3}$.

Cet article décrit la croissance par E.J.M. sur substrats de InP de couches de $\operatorname{In}_{0,53} \mathrm{Ga}_{0,47} \mathrm{As}$ accordées à mieux que $10^{-3}$ avec le substrat et caractérisées par un faible dopage résiduel.

Ces couches sont obtenues en contrôlant la préparation du substrat, la température du substrat, les flux d'indium et de gallium, la pression d'arsenic et le recuit des produits sources. La caractérisation des couches est faite par diffraction de rayons $X$, effet Hall et photoluminescence à basse température.

On montrera ensuite comment ces couches peuvent être dopées soit de type $n$, soit de type $p$ dans une large gamme $\left(10^{16} \mathrm{~cm}^{-3}-10^{19} \mathrm{~cm}^{-3}\right)$ à l'aide de l'étain et du béryllium.
Nous présentons enfin les premiers résultats obtenus sur la croissance de InP sur substrat de même nature, en employant comme sources de phosphore, soit du InP polycristallin, soit du phosphore rouge.

\section{Dispositif expérimental.}

Les expériences ont été réalisées dans un système RIBER 2300. Le vide résiduel dans la chambre d'épitaxie est inférieur à $10^{-10}$ torr. Il est obtenu par une pompe ionique, un puits à sublimation de titane, une cryopompe à bain d'hélium et une double enceinte refroidie à l'azote liquide. Pour les épitaxies de InP, l'évacuation difficile du phosphore a nécessité le montage d'une pompe turbomoléculaire; son pompage peu sélectif permet l'évacuation du phosphore.

Le manipulateur comporte un porte-substrat tournant, pour éviter les gradients de température et de composition sur la couche [3] et une jauge d'ionisation qui, placée face aux cellules avant l'épitaxie, mesure les pressions dans les flux d'indium et de gallium. Pour l'analyse in situ, nous utilisons un système de diffraction d'électrons de $10 \mathrm{kV}$ pour le contrôle de la morphologie de la surface durant la croissance et un analyseur de gaz quadripolaire. 
Un pyromètre infrarouge contrôle la température du substrat pendant l'épitaxie.

Un sas d'introduction et une chambre de préparation sont chacun pourvu d'un groupe de pompage ultravide autonome.

\section{Epitaxie par jets moléculaires de $\mathbf{I n}_{0,53} \mathbf{G a}_{0,47} A s$.}

L'épitaxie par jets moléculaires de couches $\mathrm{In}_{0,53} \mathrm{Ga}_{0,47}$ As accordées avec le substrat de InP se heurte à deux difficultés principales : la préparation du substrat et le contrôle de la composition de la couche et de sa stabilité durant l'épitaxie. Par ailleurs, en ce qui concerne les propriétés électriques des couches de $\operatorname{In}_{0,53} \mathrm{Ga}_{0,47}$ As non intentionnellement dopées, il a déjà été démontré que la température d'épitaxie et la pression d'arsenic [4] ainsi que le recuit de l'indium [5] influent sur la qualité du matériau épitaxié.

3.1 Préparation des Substrats. - La préparation de substrats de bonne planéité, exempts de défauts de polissage, est une étape primordiale pour l'élaboration de bonnes couches épitaxiées. Des traces d'oxydes ou de contaminants à la surface du substrat sont générateurs de défauts dans la couche.

Les substrats utilisés sont semi-isolants dopés-fer $\left(E P D=10^{4} \mathrm{~cm}^{-2}\right)$ et orientés $(100)$.

Notre préparation, basée sur un polissage mécanochimique à l'aide d'une solution de bromométhanol suivi d'une passivation, a été simplifiée au maximum afin d'être le plus reproductible possible.

La passivation est obtenue en immergeant le substrat dans de l'eau désionisée; il se forme alors à sa surface une fine couche d'oxyde natif (quelques dizaines d'angströms) le protégeant d'une pollution avant son introduction dans le système ultra-vide.

Pour évaporer cet oxyde, le substrat est recuit dans la chambre de préparation à $550^{\circ} \mathrm{C}$. Afin d'éviter sa dissociation thermique, une pression partielle de $10^{-3}$ torr de phosphine est nécessaire.

3. 2 ConTrôle DE LA COMPosition. - La croissance de couches de bonne qualité cristalline de $\operatorname{In}_{1-x} \mathrm{Ga}_{x} \mathrm{As}$ sur substrat de InP requiert un contrôle précis de la composition de manière à avoir une adaptation de maille avec le substrat meilleure que $10^{-3}$. L'accord de maille avec InP se faisant pour $x=0,47$, un écart de $1 \%$ autour de cette valeur entraîne un désaccord $\Delta a / a$ de $7 \times 10^{-4}$.

La composition est contrôlée par l'emploi de deux cellules séparées pour In et $\mathrm{Ga}$ dont les flux sont calibrés avant l'épitaxie à l'aide d'une jauge BayardAlpert.

\section{3 CYCLE THERMIQUE ET PRESSION D'ARSENIC PENDANT} LA CROISSANCE. - L'épitaxie doit commencer à basse température pour éviter la dissociation thermique du substrat car l'oxyde protecteur a été évaporé auparavant.

Cela permet de mieux maîtriser le début de la croissance de la couche. Après quelques dizaines de couches atomiques épitaxiées à $360^{\circ} \mathrm{C}$ et contrôlées par diffraction d'électrons (HEED), la température du substrat, $T_{\mathrm{s}}$, est augmentée progressivement jusqu'à $540^{\circ} \mathrm{C}$. Cette valeur de température semble être un compromis entre les hautes températures $\left(T_{\mathrm{s}}>560^{\circ} \mathrm{C}\right)$ pour lesquelles on observe une dissociation thermique et des pertes d'indium et des températures plus basses $\left(T_{\mathrm{s}}<530^{\circ} \mathrm{C}\right)$ pour lesquelles on note une augmentation des densités résiduelles de porteurs mesurées par effet Hall.

En début de croissance, la température est contrôlée par un pyromètre; en effet au cours d'une rampe de température, on note un décalage important entre les valeurs affichées par le pyromètre et le thermocouple de régulation, qui serait dû à la position en retrait du thermocouple.

La forte tension de vapeur de l'arsenic oblige à maintenir une pression d'As ${ }_{4}$ nettement supérieure à celle des éléments III, indium et gallium.

Nous montrons sur la figure 1 le rapport minimal des pressions $P_{\mathrm{As}} / P_{\mathrm{III}}$, nécessaire pour assurer une surface de croissance stabilisée-arsenic, en fonction de la température du substrat, $T_{\mathrm{s}}$. Ce rapport minimal est déterminé par le changement des diagrammes HEED, caractéristiques des structures stabilisée-arsenic ou stabilisée-métal [6].

Nous avons observé une amélioration sensible des propriétés électroniques, essentiellement de la mobi-

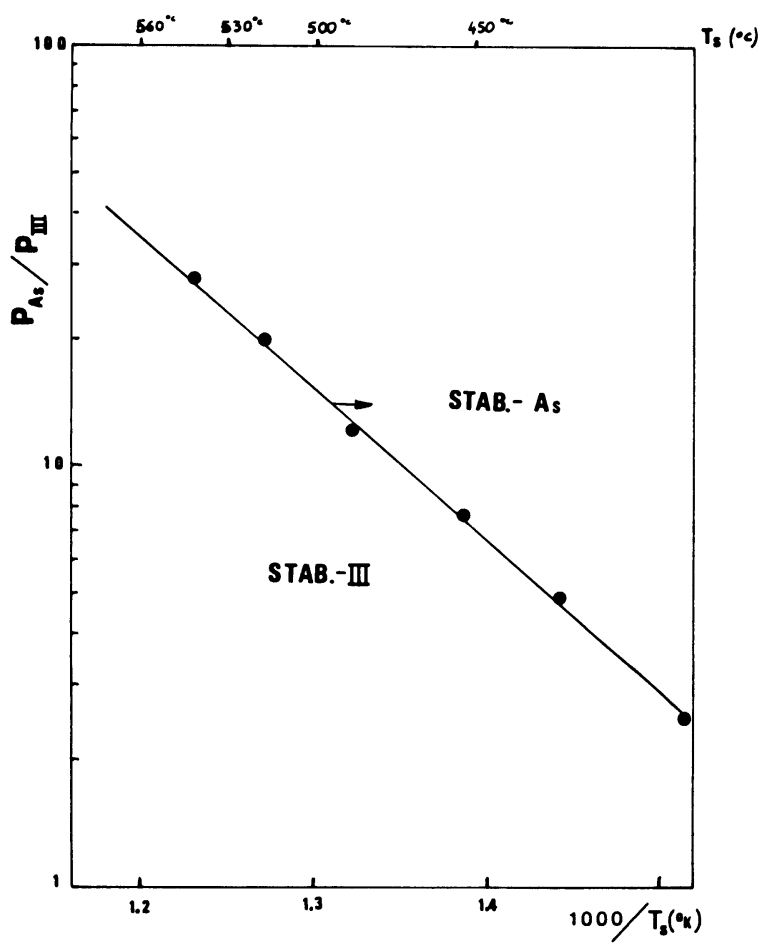

Fig. 1. - Rapport $P_{\mathrm{As}} / P_{\mathrm{In}+\mathrm{Ga}}$ limite, séparant les structures "stabilisée-arsenic " et "stabilisée-métal ", en fonction de l'inverse de la température du substrat.

[Limit pressure ratio $\mathrm{As} / \mathrm{In}+\mathrm{Ga}$ separating arsenic and metal stabilized surface structures, as a function of reciprocal substrate temperature.] 
lité, lorsque nous augmentons le rapport $P_{\text {As }} / P_{\text {III }}$ (diminution du nombre des lacunes d'arsenic).

En fait, le rapport $P_{\mathrm{As}} / P_{\text {III }}$ utilisé durant les épitaxies est typiquement deux fois supérieur au rapport limite décrit sur la figure 1 .

3.4 RECUIT DE L'INDIUM. - La pureté des produits sources utilisés est $7 \mathrm{~N}$, excepté pour les dopants, $6 \mathrm{~N}$ pour l'étain et $3 \mathrm{~N} 5$ pour le béryllium. A la lumière des résultats obtenus en épitaxie phase liquide, grâce à l'étuvage de l'indium sous hydrogène à $700{ }^{\circ} \mathrm{C}$ [5] nous appliquons le même recuit pendant $50 \mathrm{~h}$ à l'indium avant son chargement dans le réacteur.

Le silicium étant l'impureté majoritaire, ce type de traitement nous a permis de réduire sensiblement le dopage résiduel de type $n$.

\subsection{CARACTÉRISATION DES COUCHES ÉPITAXIÉES.}

3.5.1 Effet Hall. - Les mesures d'effet Hall faites sur les couches d'InGaAs non dopées, épitaxiées sur substrats semi-isolants dopés-fer mettent en évidence un dopage résiduel de type $\mathbf{n}$ caractérisé par une concentration électronique comprise entre $8 \times 10^{14}$ et $3 \times 10^{15} \mathrm{~cm}^{-3}$.

Les mobilités électroniques mesurées dans l'obscurité vont jusqu'à $11500 \mathrm{~cm}^{2} / \mathrm{V}$.s à $300 \mathrm{~K}$ et $47000 \mathrm{~cm}^{2} / \mathrm{V}$.s à $77 \mathrm{~K}$. Les valeurs moyennes se situent à $10000 \mathrm{~cm}^{2} / \mathrm{V}$.s à $300 \mathrm{~K}$ et $38000 \mathrm{~cm}^{2} / \mathrm{V}$.s à $77 \mathrm{~K}$.

La figure 2 représente les mobilités électroniques mesurées en fonction de la concentration électronique.

On constate un bon accord entre les résultats expérimentaux et le modèle théorique de Takeda [7] représenté par les lignes discontinues, qui prend en compte la diffusion de la quantité de mouvement

UNDOPED $\quad \ln _{63} \mathrm{Ga}_{47}$ As

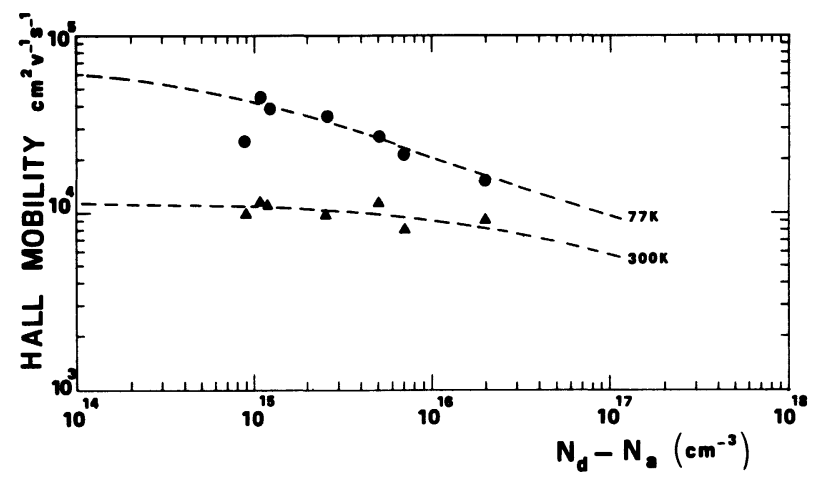

Fig. 2. - Mobilité électronique en fonction de la concentration de porteurs. Les courbes en pointillés proviennent des calculs théoriques de Takeda tenant compte d'un "alloy scattering" et d'un faible taux de compensation $\frac{N_{\mathrm{d}}+N_{\mathrm{a}}}{N_{\mathrm{d}}-N_{\mathrm{a}}}=1,5$.

[Electron hall mobility versus donor concentration. Broken lines are Takeda calculations involving alloy scattering with a slight compensation $\frac{N_{\mathrm{d}}+N_{\mathrm{a}}}{N_{\mathrm{d}}-N_{\mathrm{a}}}=1.5$.] des porteurs par un désordre d'alliage (alloy scattering), avec un taux de compensation égal à 1,5.

3.5.2 Diffraction $X$. - Le désaccord réticulaire entre la couche épitaxiale et le substrat de InP est mesuré à l'aide d'un diffractomètre simple, dont la résolution permet des mesures de $|\Delta a / a|<5 \times 10^{-4}$.

Le contrôle de la composition du ternaire assure un accord de maille des couches épitaxiales avec le substrat meilleur que $\pm 10^{-3}$.

La largeur à mi-hauteur des signaux de diffraction $\mathrm{X}$ sur les épitaxies d'InGaAs est comparable à celle mesurée sur les signaux relatifs au substrat de InP et met en évidence la bonne qualité cristalline des couches épitaxiales.

3.5.3 Photoluminescence à basse température. - Le spectre de photoluminescence à $6 \mathrm{~K}$ d'une couche de $\mathrm{In}_{0,53} \mathrm{Ga}_{0,47}$ As non intentionnellement dopée est représentée sur la figure 3 . Il présente essentiellement deux pics :

- Le premier est situé à $800 \mathrm{meV}$ et sa largeur à mi-hauteur est de $3 \mathrm{meV}$. En prenant $810 \mathrm{meV}$ comme valeur du gap à $6 \mathrm{~K}[1,8]$, il se situe à $10 \mathrm{meV}$ au-dessous. Ce pic est attribué à une transition donneur silicium-bande de valence et son élargissement aux hautes énergies est probablement dû à des transitions excitoniques non résolues [9]. La résolution de notre dispositif expérimental est de $8 \AA$.

La figure de droite donne l'intensité de ce pic en fonction de l'inverse de la température et laisse apparaître deux énergies d'activation, celle d'une recombinaison excitonique égale à $4 \mathrm{meV} \pm 0,5$ et celle d'une recombinaison donneur-bande de valence à $7 \mathrm{meV} \pm 1$. La puissance laser injectée varie de $10 \mathrm{~mW} / \mathrm{cm}^{2}$ à $40 \mathrm{~W} / \mathrm{cm}^{2}$.

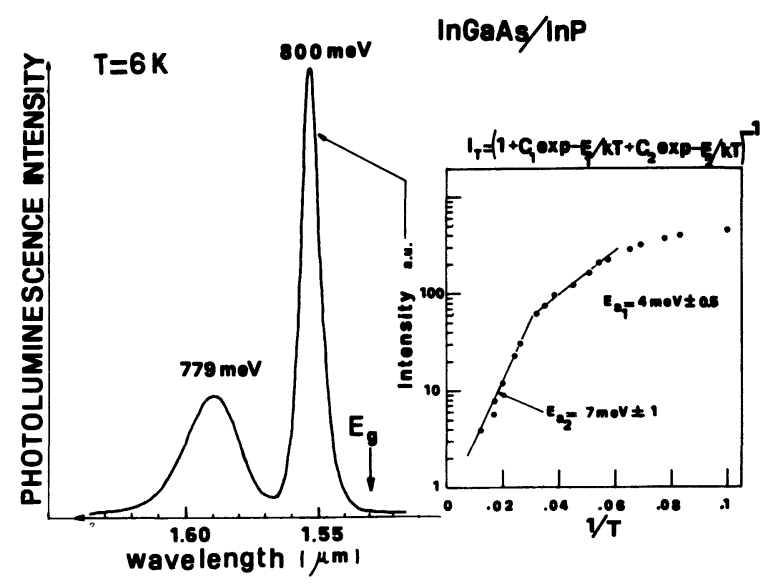

Fig. 3. - A gauche : Spectre de photoluminescence caractéristique d'une couche d'InGaAs non dopée. $A$ droite : l'intensité du $1^{\mathrm{er}}$ pic en fonction de $1 / T ; 2$ énergies d'activation sont déterminées à $4 \mathrm{meV}$ et $7 \mathrm{meV}$.

[Typical photoluminescence spectrum of undoped MBE InGaAs(left). Integrated intensity of the first line as a function of reciprocal temperature (right); two activation energies are determined, $4 \mathrm{meV}$ and $7 \mathrm{meV}$.] 
— Le second pic situé vers $780 \mathrm{meV}$ présente les caractéristiques suivantes :

- Déplacement vers les hautes énergies avec l'augmentation de la concentration d'accepteurs, ainsi qu'avec l'augmentation de la puissance laser injectée.

- Décroissance rapide de l'intensité du pic et déplacement vers les hautes énergies lorsque l'on augmente la température de $6 \mathrm{~K}$ à $35 \mathrm{~K}$.

- L'amplitude du pic est une fonction croissante du niveau de carbone résiduel détecté dans le réacteur à l'aide d'un spectromètre de masse.

Compte tenu des observations suivantes ce pic est attribué à une recombinaison de paires donneuraccepteur silicium-carbone, dont nous tirons une énergie d'ionisation du carbone accepteur $E_{\mathrm{i}}=19 \mathrm{meV}$.

Cette recombinaison de paires $\mathrm{D}-\mathrm{A}$ a déjà été observée dans le matériau ternaire InGaAs épitaxié en phase liquide [9] et en phase gazeuse [8].

3.6 Dopages. - Nous présentons les résultats obtenus sur des couches de $\operatorname{In}_{0,53} \mathrm{Ga}_{0,47} \mathrm{As}$ dopées à l'étain et au béryllium. Ces dopants à faible coefficient de diffusion, permettent la réalisation de profils de dopage ou de jonctions très abrupts.

Les niveaux de dopages sont contrôlés de façon reproductible entre $10^{16} \mathrm{~cm}^{-3}$ et $10^{19} \mathrm{~cm}^{-3}$ sans qu'un changement de morphologie ne soit observé pendant la croissance.

La figure 4 donne les concentrations de porteurs mesurées par effet Hall en foncicicn de la température de la cellule d'étain (surface d'ouverture de la

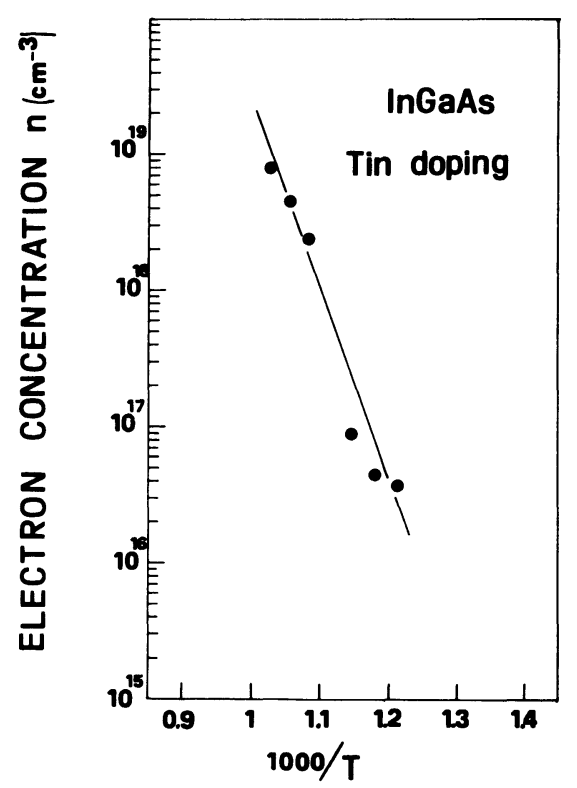

Fig. 4. - Concentration électronique des couches InGaAs dopées à l'étain en fonction de la température de la source d'étain.

[Electron concentration in tin doped InGaAs layers as a function of tin source temperature.] cellule $=3 \mathrm{~cm}^{2}$, distance par rapport au substrat $=$ $12 \mathrm{~cm}$, vitesse de croissance $=1,8 \mu \mathrm{m} / \mathrm{h}$ ).

Les mobilités des couches dopées type n, en fonction de la concentration électronique montrent un bon accord avec le calcul de Takeda [7].

La figure 5 montre la concentration de trous des couches de InGaAs dopées-béryllium en fonction de la température de la source. Des niveaux de dopage allant jusqu'à $10^{19} \mathrm{~cm}^{-3}$ ne modifient pas la morphologie de surface contrôlée par diffraction HEED. En revanche, il semble que des concentrations supérieures à $10^{19} \mathrm{~cm}^{-3}$ entraînent une modification de morphologie de la surface de croissance.

\section{Epitaxie de InP sur substrat de InP.}

L'épitaxie du matériau binarre InP par jets moléculaires n'a été abordée jusqu'ici que par un petit nombre de laboratoires [10-13].

Le problème majeur lié à la croissance du phosphure d'indium par E.J.M. est la présence de fortes pressions de phosphore dont l'élimination reste difficile après épitaxie.

Nous présentons ici des résultats préliminaires sur l'épitaxie de InP par E.J.M.

Deux types de sources de phosphore ont été utilisées, le phosphure d'indium polycristallin de haute pureté $\left(n=5 \times 10^{15} \mathrm{~cm}^{-3}\right)$ et le phosphore rouge de pureté $6 \mathrm{~N}$.

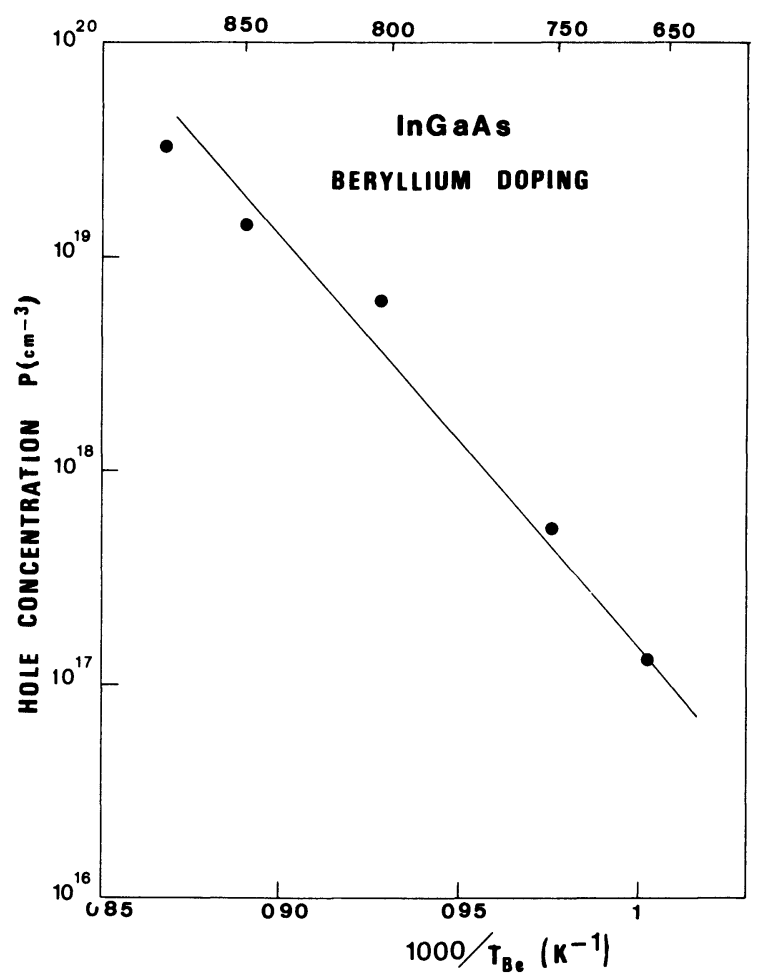

Fig. 5. - Concentration de trous des couches InGaAs dopées au béryllium en fonction de la température de la source de béryllium.

[Hole concentration in beryllium doped InGaAs layers, as a function beryllium source temperature.] 
L'utilisation du phosphure d'indium polycristallin présente l'inconvénient d'un appauvrissement rapide en phosphore obligeant à une élévation presque constante de la température de la source entre $300^{\circ} \mathrm{C}$ et $800{ }^{\circ} \mathrm{C}$ pour conserver un flux de phosphore $\mathrm{P}_{2}$ constant. Bien que la tension de vapeur de $\mathrm{P}_{2}$ soit plus faible que celle de $\mathrm{P}_{4}$, cet appauvrissement de la source est suffisamment rapide pour ne permettre que la croissance de couches de 4 microns d'épaisseur avec un creuset de type CBN $125\left(\sim 20 \mathrm{~cm}^{3}\right)$. Les couches épitaxiées dans ces conditions présentent une bonne qualité cristalline mais le dopage résiduel reste élevé $\left(n>5 \times 10^{17} \mathrm{~cm}^{-3}\right)$. Celui-ci est probablement dû aux impuretés présentes dans le phosphure d'indium polycristallin.

Le deuxième type de source phosphore a été la sublimation du phosphore rouge en molécules tétramères $\mathrm{P}_{4}$

Comme dans le cas du ternaire $\mathrm{In}_{0,53} \mathrm{Ga}_{0,47} \mathrm{As}$, nous avons déterminé le rapport du flux de phosphore $\mathrm{P}_{4}$ sur le flux d'indium, nécessaire pour obtenir une surface de croissance stabilisée-phosphore pendant l'épitaxie. Le rapport de pressions $P_{\mathrm{P}} / P_{\mathrm{In}}$ mesurées doit être supérieur ou égal à 200 (soit 50 pour $P_{\mathrm{P}_{4}} / P_{\mathrm{In}}$ ) pour une température de substrat $T_{\mathrm{s}}$ égale à $500^{\circ} \mathrm{C}$. Le faible "coefficient de collage apparent " du phosphore $\mathrm{P}_{4}$ entraîne une forte consommation du produit source qui limite les épitaxies à des épaisseurs de l'ordre de $5 \mu \mathrm{m}$ avec un creuset PBN 125.

Les mesures de diffraction $\mathrm{X}$ indiquent une bonne qualité cristalline des monocouches de InP épitaxiées par cette deuxième technique. Pour des températures de croissance situées entre $450^{\circ} \mathrm{C}$ et $530^{\circ} \mathrm{C}$, le dopage résiduel obtenu varie entre $5 \times 10^{15} \mathrm{~cm}^{-3}$ et $10^{16} \mathrm{~cm}^{-3}$. La mobilité électronique égale $1500 \mathrm{~cm}^{2} / \mathrm{V}$.s à $300 \mathrm{~K}$ indique une compensation relativement élevée. En effet, avant l'épitaxie, il est indispensable d'effectuer un dégazage in situ du phosphore rouge qui adsorbe en quantité importante $\mathrm{H}_{2} \mathrm{O}$ et en quantité moindre le $\mathrm{CO}$. Ce dégazage est effectué à des températures peu élevées pendant plusieurs dizaines d'heures.
Le rapport ${ }^{18} \mathrm{H}_{2} \mathrm{O}^{+} /{ }^{124} \mathrm{P}_{4}^{+}$est contrôlé au spectromètre de masse. Une meilleure détermination des conditions de désorption de ces impuretés devrait nous permettre de réduire sensiblement le dopage résiduel.

\section{Conclusion.}

Par une préparation efficace des substrats, un contrôle précis des flux de gallium et d'indium et de la température du substrat, ainsi qu'une pression suffisante d'arsenic, nous obtenons des couches de ternaire $\mathrm{In}_{0,53} \mathrm{Ga}_{0,47} \mathrm{As}$ de haute pureté. Le désaccord réticulaire entre la couche de ternaire et le substrat est inférieur à $10^{-3}$.

Les couches non dopées sont de type $\mathrm{n}$ avec une concentration de porteurs comprise entre $8 \times 10^{14} \mathrm{~cm}^{-3}$ et $3 \times 10^{15} \mathrm{~cm}^{-3}$ et des mobilités électroniques à $300 \mathrm{~K}$ et $77 \mathrm{~K}$ allant respectivement jusqu'à $11500 \mathrm{~cm}^{2} /$ V.s et $47000 \mathrm{~cm}^{2} /$ V.s. Les dopages de type $\mathrm{n}$ à l'étain et de type $\mathrm{p}$ au béryllium sont facilement contrôlés de $10^{16}$ à $10^{19} \mathrm{~cm}^{-3}$.

Enfin, l'épitaxie de monocouches de InP en utilisant, soit le phosphure d'indium polycristallin, soit le phosphore rouge sous la forme tétramère, a été réalisée avec de bonnes qualités cristallines. Le dopage résiduel allant de $5 \times 10^{15} \mathrm{~cm}^{-3}$ à $10^{16} \mathrm{~cm}^{-3}$ avec une mobilité $\mu_{300 \mathrm{~K}}=1500 \mathrm{~cm}^{2} / \mathrm{V}$.s indique une compensation que nous attribuons aux impuretés adsorbées par le phosphore rouge. Bien que ces deux types de sources de phosphore aient une faible durée de vie, ces premières épitaxies de InP nous ont permis de résoudre les problèmes techniques assurant la maîtrise du phosphore.

\section{Remerciements.}

Les auteurs remercient $\mathrm{D}$. Bonnevie pour sa contribution expérimentale, C. Audry pour les mesures de diffraction X, A. Perronnet pour la caractérisation et J. Benoit pour ses conseils et discussions enrichissants.

Bibliographie

[1] Pearsall, T. P., IEEE J. Quantum Electron. 16 (1980) 709.

[2] Ohno, H., Barnard, J., Wood, C. E. C., Eastman, L. F., IEEE Trans. Electron. Devices Lett. 8 (1980) 156.

[3] Cheng, K. Y., Cho, A. Y., Wagner, W. R., Appl. Phys. Lett. 39 (8) (1981) 607.

[4] Cheng, K. Y., Сно, A. Y., Collected paper of M.B.E.CST-2 Tokyo 1982

[5] Vergnaud, R. et al. " Journées Nationales des Semiconducteurs Composés III-V » Toulouse 1983.

[6] Chо, A. Y., Surf. Sci. 17 (1969) 494.

[7] TAKeda, Y., SaSaki, A., Jpn. J. Appl. Phys. 19 (1980) 283.
[8] Goetz, K. H., Solomonov, A. V., Bimberg, D., Jüngensen, H., Razeghi, M., J. Physique Colloq. 43 (1982) C5-383.

[9] Chen, Yu-Ssu, Kıм, O. K., J. Appl. Phys. 52 (12) (1981) 7392.

[10] Farrow, R. F. C., J. Phys. D 7 (1974) L 121.

[11] McFee, J. H., Miller, B. I., BachmanN, K. J., J. Electrochem. Soc. 124 (2) (1977) 259.

[12] Asahi, H., Kawamura, Y., IKeda, M., Окamoto, H., J. Appl. Phys. 52 (4) (1981) 2852.

[13] Tsang, W. T., Miller, R. C., Capasso, F., Bonner, W. A., Appl. Phys. Lett. 41 (5) (1982) 467. 